FORMATION Formation emploi

Revue française de sciences sociales

100 | octobre-décembre 2007

De la formation professionnelle en Suisse

\title{
La qualification ouvrière en Suisse : vers un système à deux vitesses ?
}

Worker qualification in Switzerland: a dual system responds to change Facharbeiterausbildung in der Schweiz: bald ein System mit zwei Gangarten

Laurence Marti

\section{OpenEdition}

Journals

Édition électronique

URL : http://journals.openedition.org/formationemploi/1260

DOI : 10.4000/formationemploi.1260

ISSN : 2107-0946

Éditeur

La Documentation française

Édition imprimée

Date de publication : 1 octobre 2007

Pagination : $31-47$

ISSN : 0759-6340

Référence électronique

Laurence Marti, "La qualification ouvrière en Suisse : vers un système à deux vitesses ? », Formation emploi [En ligne], 100 | octobre-décembre 2007, mis en ligne le 01 octobre 2009, consulté le 30 octobre 2020. URL : http://journals.openedition.org/formationemploi/1260 ; DOI : https://doi.org/ 10.4000/formationemploi.1260

(c) Tous droits réservés 


\title{
DOSSIER
}

\section{La qualification ouvrière en Suisse : vers un système à deux vitesses ? 1}

\author{
Par Laurence Marti*
}

\author{
Le processus adopté par la Suisse en matière de qualification ouvrière, \\ en apparence fidèle au principe indéfectible de l'apprentissage, ne manque pas \\ d'être traversé par des mouvements qui le remettent en partie en question \\ et en modifient les contours.
}

Depuis quelques années, la question de la qualification dans les métiers ouvriers fait l'objet en Suisse d'un important débat, lié aux transformations qui touchent aujourd'hui le monde du travail et de l'emploi ouvriers. Le terme de qualification est entendu ici, non comme un donné de « nature », mais comme le résultat d'un processus négocié visant à mettre en relation des potentialités reconnues à la force de travail et les tâches qui lui sont attribuées (Voir Paradeise, 1988 ou Méda et Vennat, 2004). Parmi les transformations observées, il faut évoquer bien sûr celles qui affectent les contenus du travail (détaylorisation, prise d'importance des compétences relationnelles sur les compétences techniques, accent mis sur la polyvalence et la flexibilité, etc.). Elles ont

\footnotetext{
${ }^{1}$ L'article est issu de deux recherches récentes :

1. Un projet financé par le Fonds national de la recherche scientifique dans le cadre du Programme national de recherche « Formation et emploi » (PNR 43);

2. Une recherche menée entre 2002 et 2004 sur la vie des mécaniciens aujourd'hui et réalisée grâce à un financement privé. Cette recherche a fait l'objet d'une publication (Marti et al., 2005).
}

conduit à une redéfinition souvent conséquente des compétences nécessaires à l'exercice des métiers et à une hausse des exigences. Mais il faut également citer les transformations qui touchent aux processus d'acquisition de la connaissance. L'introduction, dans la nouvelle loi sur la formation professionnelle de 2002 , de procédures de validation d'acquis susceptibles de répondre à une mobilité professionnelle de plus en plus importante, mais aussi à la reconversion d'ouvriers victimes du chômage, bouscule les processus historiques d'apprentissage basés sur une formation acquise principalement en début de vie

* Laurence Marti est docteur en sociologie et historienne. Elle dirige un bureau de recherches indépendant près de Lausanne. Ses travaux sont centrés sur la sociologie et l'histoire de l'entreprise et du travail, plus particulièrement du travail ouvrier. Elle a publié plusieurs ouvrages dont L'invention de l'horloger (2003), Éditions Antipodes, et récemment en collaboration avec F. Messant et M. Modak (2005), Vies de mécaniciens, Éditions Antipodes. 
professionnelle. Enfin, le statut même de l'emploi et $\mathrm{du}$ travail ouvriers est lui aussi en évolution. La désaffection des jeunes pour les métiers ouvriers est en voie de provoquer une pénurie d'ouvriers et ouvrières qualifiés dans de nombreux secteurs, et ce malgré la diminution progressive des emplois ouvriers; ce phénomène conduit les collectivités publiques et les entreprises à prendre des mesures de revalorisation de la formation ouvrière et à trouver des processus différents et plus rapides d'accès à la qualification.

Ces transformations n'ont pas manqué d'interpeller les différents acteurs de la formation professionnelle et de susciter le débat sur la pertinence du maintien du modèle helvétique d'acquisition de la qualification ouvrière. Celui-ci, rappelons-le, repose historiquement d'une part sur le suivi d'un apprentissage de type dual, proche du modèle allemand, durant lequel l'apprenant partage son temps hebdomadaire de formation entre une pratique en entreprise et le suivi de cours en école professionnelle, et d'autre part sur la détention d'un titre reconnu, le certificat fédéral de capacité (CFC). Les origines de ce modèle remontent au moins au début de la législation sur la formation professionnelle, soit aux années 30 , mais on peut y voir un héritage plus ancien : celui des corporations, puis des associations de métier, encore très actives et puissantes à la fin du XIX ${ }^{e}$ siècle. L'entrée en vigueur de la loi de 1930 avait ainsi été saluée comme «un retour aux principes économiques qui ont disparu avec les corporations après avoir fait la force des métiers du Moyen Âge. [...] Cette loi consacre précisément la restauration de la corporation qui remettra en honneur l'apprentissage en tenant compte de l'évolution économique» (Tabin, 1984, p. 82). Cette conception n'a jamais subi de remise en question fondamentale. Les grilles à critères classants $^{2}$, chères à la métallurgie française, n'ont par exemple jamais été introduites en Suisse.

\footnotetext{
${ }^{2}$ Les grilles à critères classants ont été introduites en France dans les années 70. Les niveaux de qualification du personnel sont établis non pas sur la base d'une tâche générale à accomplir ou sur le diplôme, mais sur les compétences, notamment les aptitudes et capacités comportementales (autonomie, initiative, etc.), mises en œuvre effectivement dans le cadre du travail. Chaque niveau regroupe les postes d'une entreprise ou d'une branche nécessitant la mise en œuvre des mêmes compétences. L'introduction de ces grilles est souvent considérée comme une première forme d'internalisation et d'individualisation des processus de qualification.
}

Ainsi, le suivi de l'apprentissage et son corollaire, le CFC, ont figuré au cœur de la définition de la qualification ouvrière durant tout le $\mathrm{XX}^{\mathrm{e}}$ siècle et ont servi de base pour la classification des ouvriers en deux catégories, qualifiés et non-qualifiés, selon qu'ils étaient ou non détenteurs d'un CFC.

En Suisse, le marché du travail ouvrier s'est donc structuré davantage selon une logique de marché de métier ou professionnel que selon une logique de marché interne ou d'entreprise, pour reprendre la distinction de D. Marsden (1989). En effet, si l'apprentissage est réalisé partiellement en entreprise, son contenu et son déroulement sont définis hors de l'entreprise par les partenaires sociaux et l'État qui interviennent à tous les niveaux de décision. Globalement, l'accent a été mis sur la transférabilité des connaissances et sur une protection de l'accès aux emplois professionnels qui sont longtemps restés réservés aux seuls détenteurs d'un CFC. La mobilité verticale des «non-qualifiés » en a ainsi été fortement freinée ${ }^{3}$.

Quelles sont aujourd'hui les réponses apportées aux transformations subies par ce système? Les décisions prises à l'échelle nationale en matière de politique de formation professionnelle ont surtout été marquées par une réaffirmation du bien-fondé de l'apprentissage sous sa forme duale. La nouvelle loi sur la formation professionnelle de 2002, entrée en vigueur en 2004, va dans ce sens ${ }^{4}$.

Le maintien des principes de base a néanmoins été complété par plusieurs mesures d'extension, de renforcement et de revalorisation. La création de réseaux de formation, soit la mise en commun de ressources de formation entre entreprises, permet désormais à de petites entreprises d'offrir une formation complète à un apprenti. On parle dans ce cas de formation triale. Le contenu des apprentissages est

\footnotetext{
${ }^{3}$ Dans un marché interne ou d'entreprise, la définition et le contrôle des processus de qualification et d'accès aux postes de travail sont le fait d'une entreprise et non d'une profession. Il en résulte, entre autres, une certaine ouverture en termes de mobilité interne, mais une non-transférabilité des savoirs.

${ }^{4}$ Le Conseil fédéral le réaffirme dans son message de 2000 : " L'apprentissage s'est révélé une excellente voie d'insertion dans la vie professionnelle et une méthode efficace d'acquisition des connaissances. Il reste par conséquent le pilier central de la formation professionnelle. » (Message du Conseil Fédéral, 2000, p. 5528)
} 
aussi progressivement revu afin de l'adapter aux nouvelles exigences du travail ouvrier. La revalorisation s'est également traduite par la mise en place de passerelles conduisant du CFC à des niveaux de formation supérieurs, la maturité (bac) notamment, et au-delà jusqu'à un niveau universitaire ou une école polytechnique. Enfin, plusieurs campagnes de sensibilisation ont été lancées. D'abord auprès des jeunes, notamment des filles, pour les encourager à suivre cette voie de formation. Ensuite auprès des entreprises pour les amener à créer davantage de places d'apprentissage, l'offre actuelle ne suffisant pas à combler la demandes.

Ce mouvement est largement soutenu par les partenaires sociaux. L'association faîtière de l'industrie des machines a, par exemple, établi à l'intention de ses membres une liste des «sept bonnes raisons de former des apprentis $»^{6}$.

De toutes parts donc, l'engagement en faveur du maintien et du développement de cette voie de formation a été manifeste. La préférence est allée à une revalorisation et à une extension de l'apprentissage plutôt qu'à un encouragement à suivre des formations supérieures. Tout converge donc pour que l'apprentissage garde, voire augmente sa position dominante en matière de qualification ouvrière. Cette filière reste de fait la plus suivie, puisque $75 \%$ des jeunes optent aujourd'hui pour un apprentissage, dont une majorité sous forme duale (graphique 1).

\section{Graphique 1 \\ Type d'apprentissage suivi par les personnes ayant commencé une formation professionnelle} (Source : OFS)

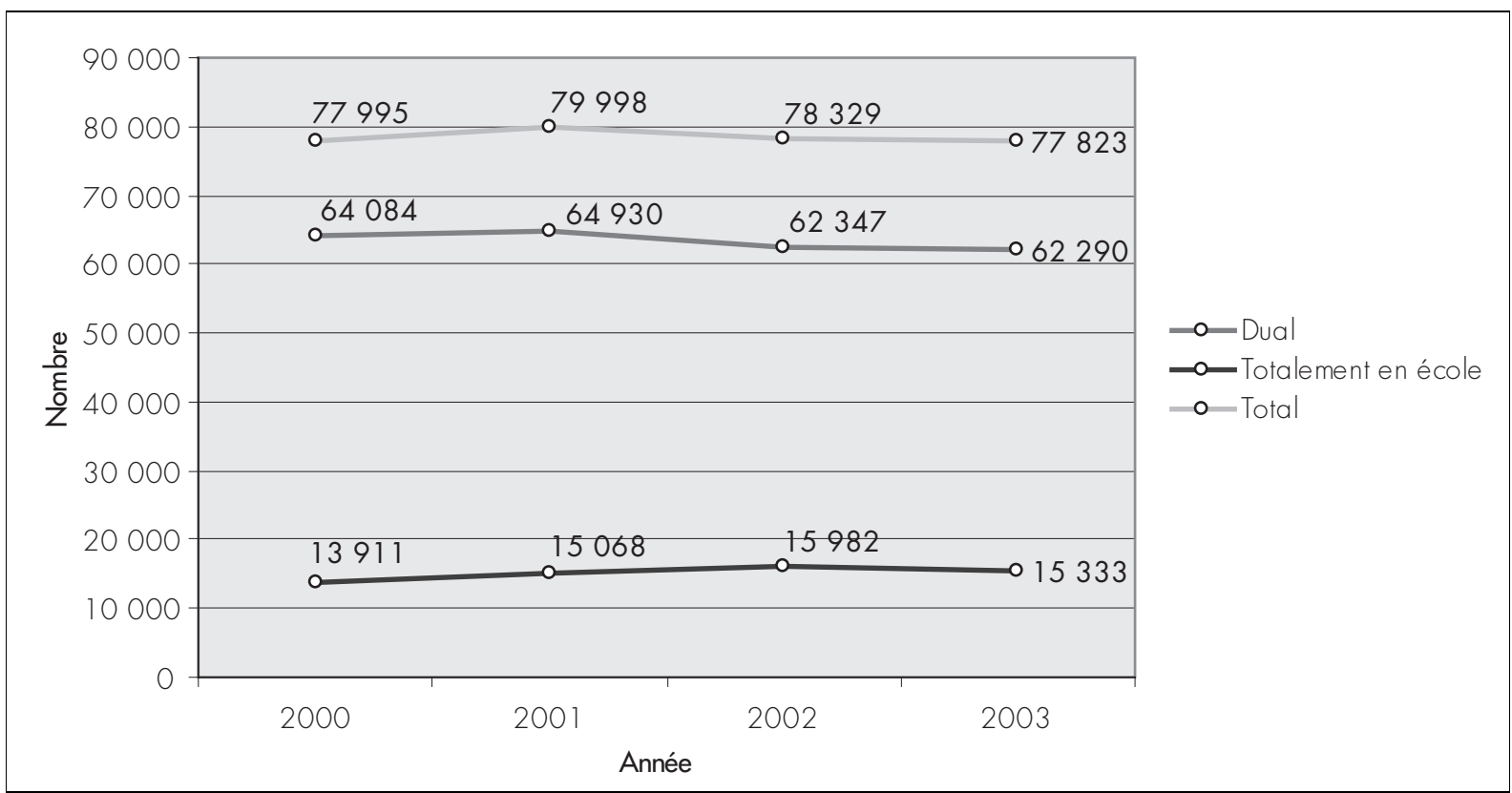

5 Pour plus de détails sur la situation actuelle en matière de politique de formation professionnelle, voir Dubs R. (2006), Rapport d'expertise sur les questions concernant la formation professionnelle en Suisse, Hep Verlag, Berne.

6 Parmi ces raisons, on peut lire : "Pour chaque entreprise, la compétence des collaboratrices et des collaborateurs est un facteur de succès décisif. La formation d'une relève professionnelle qualifiée assure la compétitivité ainsi que l'avenir de l'entreprise et de la branche » ou encore « La formation professionnelle reposant sur deux ou trois voies est qualitativement meilleure, répond mieux aux besoins, est plus souple et économique qu'un système purement scolaire. Avec la formation d'apprentis, les entreprises assurent la pérennité du système de formation professionnelle suisse. De ce fait, elles endossent une responsabilité sociale. » Swissmem (2005), Sept bonnes raisons de former des apprentis, site www. swissmem.ch 
En apparence, ce processus ne laisse donc guère de place à d'autres formes d'acquisition de la qualification. En réalité, ce n'est pas tout à fait le cas. L'ancienne loi suisse sur la formation professionnelle de 1978, au travers de son article 41, prévoyait la possibilité pour des adultes de se présenter, en cours de carrière, au CFC, moyennant quelques conditions préalables (un nombre d'années d'expérience, le suivi de cours, etc.). Ainsi, quelque $5 \%$ des apprentis suivaient cette voie de formation chaque année.

Si ce dernier type de filière a peu été investi par le monde ouvrier et finalement par les non-diplômés, comme le montrent les analyses de R. Schräder-Naef (1997 et 2003), son existence assurait une certaine souplesse par rapport au principe de l'apprentissage initial et laissait une chance d'être reconnus à ceux qui n'avaient pas pu en profiter. La nouvelle loi de 2002 a conservé cette possibilité, en remplaçant son fameux article 41 par un ensemble de dispositions favorisant des filières de formation nouvelles, telles que la validation des acquis. Ces filières sont aujourd'hui en phase d'institutionnalisation au niveau de chaque canton et pourraient modifier plus fondamentalement les rythmes et les temps d'acquisition.

À l'échelle des entreprises, certaines ouvertures sont aussi intervenues, mettant davantage l'accent sur la formation interne. Durant les années 1950-1970, pour pallier le manque de main-d'œuvre qualifiée, des personnes non diplômées ont pu accéder, moyennant une formation réalisée «sur le tas", à des postes réservés en principe à des diplômés. On a aussi vu fleurir l'expression de «semi-qualifiés", pour qualifier justement ces personnes dont l'expérience pratique en emploi permettait de combler l'absence de CFC. On verra plus bas que ce genre d'accès à la qualification dans un contexte où la pénurie de main-d'œuvre qualifiée est à nouveau à l'ordre du jour, se retrouve aujourd'hui encore sous de nouvelles formes.

Ces constats ne font que rappeler qu'un principe central, en l'occurrence l'importance accordée à l'apprentissage, est susceptible de réinterprétations multiples dans la pratique. C'est vrai dans la comparaison de contextes nationaux (cf. par ex. M. Maurice et alii, 1982), c'est vrai aussi à l'intérieur d'un même espace de référence. La remarque vaut tout particuliè- rement pour la Suisse, qui connaît une structure sociopolitique et économique totalement décentralisée.

Les acteurs sont ainsi différents d'un secteur d'activité à l'autre et d'une région à l'autre, sans parler des spécificités propres à chaque branche, que cela soit en termes culturels, techniques ou économiques. Aborder le thème de la qualification sans tenir compte de ces différences serait par conséquent se risquer à des généralisations hâtives et pourrait compromettre la compréhension du fonctionnement concret des processus de qualification. Malheureusement, cette approche n'a pas été beaucoup explorée par les chercheurs suisses. Il n'existe pas, par exemple, d'études quantitatives qui permettraient de saisir l'importance respective des différents processus de qualification et des changements intervenus dans ce domaine à l'échelle des entreprises ou des différents secteurs. Certains processus sont par ailleurs d'origine trop récente pour qu'on puisse en mesurer l'impact réel. En nous appuyant sur nos travaux menés depuis quelques années sur le travail et l'emploi ouvriers, il est néanmoins possible de présenter un premier état des lieux sur les tendances en présence.

Cet article porte plus particulièrement sur les observations réalisées dans trois secteurs d'activité : la restauration, la fabrication de machines et l'horlogerie, qui sont porteurs, comme nous le verrons plus loin, d'une certaine tradition en matière de qualification ouvrière, tout en restant aujourd'hui encore des secteurs dynamiques en matière d'emploi. L'intérêt d'un tel choix nous paraît résider dans la possibilité de saisir jusqu'à quel point ces secteurs, a priori plutôt attachés à un modèle historique d'acquisition de la qualification, sont eux aussi amenés à procéder à certains réaménagements.

Quelles reconfigurations y sont à l'œuvre aujourd'hui en matière de qualification, quelle place est accordée à des processus alternatifs et comment y sont-ils considérés ? L'analyse proposée ici s'articule autour de ces questions. Elle s'attache plus particulièrement à mettre en évidence la diversité effective des processus de qualification développés sur le terrain des entreprises, sur la transformation et l'émergence de nouvelles formes de qualifications et sur les enjeux en termes de statut de ces nouvelles formations. 


\section{Encadré 1 \\ Le résultat d'un ensemble de recherches}

Cet article repose sur un ensemble de travaux sociologiques et historiques autour du travail ouvrier, dans trois secteurs spécifiques : la restauration, la fabrication de machines et l'horlogerie. La réflexion présentée ici s'inspire surtout de trois recherches:

- une recherche menée sur le thème de la formation professionnelle continue des ouvriers qualifiés. Elle a consisté en une soixantaine d'entretiens semi-directifs réalisés en 2001 auprès d'hommes et de femmes issus des professions d'horloger-ère et de cuisinier-ère et travaillant respectivement dans une entreprise privée de la branche horlogère et dans une entreprise publique de restauration collective. Les questions portaient sur les formations professionnelles et continues suivies, sur la conception de la formation continue, sur les perspectives d'emploi ou encore sur la mobilité interne. Ces entretiens ont été complétés par une analyse des politiques de l'entreprise en matière de formation et par des entretiens avec les responsables de la formation interne. Elle a été financée par le Fonds national de la recherche scientifique dans le cadre de son programme national de recherche "Formation et emploi » (PNR 41), (FNRS, 2004). - une recherche sur la vie et le statut des mécaniciens qualifiés aujourd'hui. Pour cette étude, une vingtaine d'entretiens approfondis ont été réalisés auprès de mécaniciens travaillant dans une entreprise de fabrication de machines-outils, associés à une recherche historique sur l'évolution de la branche et du métier. Les thèmes abordés concernaient l'évolution du contenu du travail, la qualification, l'évolution des entreprises et plus largement les changements intervenus dans la vie ouvrière au sein d'une région à tradition industrielle (Marti et al., 2005).

- un travail de mise au point d'un processus pilote de reconnaissance des acquis (projet Femhor) dans la branche horlogère qui a impliqué l'observation en situation de travail d'une centaine d'ouvriers-ères non qualifiés ou semi-qualifiés. Les observations réalisées en atelier étaient de l'ordre de quelques heures, le temps pour ces personnes de réaliser un processus de travail complet. Une trentaine d'entretiens approfondis avec ces ouvriers et des entretiens complémentaires avec les responsables ressources humaines des entreprises concernées et les partenaires sociaux ont également été réalisés. Ils portaient sur l'évolution du travail, sur la formation, la " carrière », le rapport au travail et les perspectives de mobilité. Cette étude a été soutenue par le Bureau fédéral de l'égalité entre femmes et hommes et les partenaires sociaux (Marti, 2003).

\section{TROIS SECTEURS QUI INCARNENT LA TRADITION DANS LA QUALIFICATION OUVRIÈRE}

Ces trois secteurs présentent plusieurs points communs. Ils partagent des origines qui remontent au moins au XIXe siècle et les principales professions qui y sont associées, cuisinier, horloger, mécanicien ont vu leur règlement respectif établi durant les années 1930-1940, soit dès les débuts de la réglementation fédérale sur la formation professionnelle qui date de 1930. Ils incarnent donc une certaine tradition dans la qualification ouvrière. Les trois secteurs sont également importants en matière d'emplois à l'échelle nationale. L'horlogerie et la fabrication de machines sont les deux plus grands employeurs dans le secteur des industries manufacturières, alors que la restauration figure parmi les plus grands employeurs du secteur tertiaire ${ }^{7}$ et probablement le plus grand (les chiffres manquent pour le vérifier) en matière de recrutement ouvrier dans ce secteur. Les professions de cuisinier et de mécanicien figurent parmi les professions ouvrières les plus choisies à l'échelle nationale, alors que le métier

7187000 emplois en 2006 pour les machines et l'horlogerie/bijouterie, correspondant au quart des emplois manufacturiers. 246000 en 2006 pour la restauration et l'hôtellerie, soit $8 \%$ des emplois tertiaires. Source : Office fédéral de la statistique. Indicateurs du marché de l'emploi. 
Tableau 1

Les vingt formations en apprentissage les plus fréquemment choisies en 2004 (*)

\begin{tabular}{|c|c|c|}
\hline & Formation & Nombre d'apprentis-es \\
\hline 1 & Employé-e de commerce & 11617 \\
\hline 2 & Vendeur-euse & 4186 \\
\hline 3 & École supérieure de commerce & 4029 \\
\hline 4 & Gestionnaire de vente & 2760 \\
\hline 5 & Monteur-électricien & 2271 \\
\hline 6 & Cuisinier-ère & 2090 \\
\hline 7 & Polymécanicien-ne & 1849 \\
\hline 8 & Coiffeur-euse & 1761 \\
\hline 9 & Menuisier-ébéniste & 1618 \\
\hline 10 & Mécanicien-ne d'automobiles & 1597 \\
\hline 11 & Informaticien-ne & 1584 \\
\hline 12 & Assistantee en soins et santé communautaire & 1396 \\
\hline 13 & Jardinier & 1282 \\
\hline 14 & Maçon & 1241 \\
\hline 15 & Réparateur-rice d'automobiles & 1163 \\
\hline 16 & Aide soignant-e & 1124 \\
\hline 17 & Peintre & 1029 \\
\hline 18 & Charpentier & 982 \\
\hline 19 & Monteur-euse sanitaire & 950 \\
\hline \multirow[t]{3}{*}{20} & Assistant-e médical-e & 938 \\
\hline & Total sur les vingt premières formations & 45467 \\
\hline & Nombre total d'apprenti-es & 70761 \\
\hline
\end{tabular}

$(*)$ : La profession d'horloger intervient plus bas dans le classement avec 170 apprenti-es.

Source : Office fédéral de la statistique.

d'horloger reste une référence à l'échelle régionale pour tout l'arc jurassien, où cette industrie est implantée, soit la région qui s'étend de Genève à Bâle tout le long de la chaîne du Jura (tableau 1).

Enfin, les trois secteurs connaissent une situation de pénurie de main-d'œuvre qualifiée plutôt supérieure à la moyenne des secteurs secondaire ou tertiaire correspondants (sauf pour la fabrication de machines et d'équipements) et un excédent de main-d'œuvre non qualifiée très élevé (tableau 2). Une situation qui s'avère particulièrement propice à une observation sur l'évolution du processus de qualification.

En même temps, au-delà de ces similitudes, la fabrication de machines et l'horlogerie se rattachent au secteur secondaire et connaissent un déclin de leurs effectifs, alors que la restauration, inscrite dans le secteur tertiaire, voire dans le service public, connaît une hausse de ses effectifs et participe de la dynamique générale de ce secteur. Les professions et les cultures professionnelles ont également subi des évolutions différentes : proches encore du «métier» au sens artisanal du terme dans le cadre de l'horlogerie et de la restauration, elles sont totalement transformées dans le cadre de la mécanique. La toute nouvelle profession de polymécanicien suppose désormais l'acquisition, certes prioritaire, de savoirfaire, mais s'y ajoute également un ensemble de compétences nouvelles : «La multitude des moyens de production, outils, accessoires et méthodes, de même que leur évolution rapide, exigent de nouvelles qualifications de la part des professionnels de demain. La recherche de personnel ne donne plus 
Tableau 2

Part des établissements annonçant une pénurie ou un excédent de main-d'œuvre (\%)

\begin{tabular}{|l|c|c|}
\hline \multicolumn{1}{|c|}{2003} & $\begin{array}{c}\text { Pénurie de main-d'œuvre } \\
\text { qualifiée }\end{array}$ & $\begin{array}{c}\text { Excédent de main-d'œuvre } \\
\text { non qualifiée }\end{array}$ \\
\hline Secteur secondaire & $\mathbf{1 8}$ & $\mathbf{2 7}$ \\
\hline Fabrication de machines et d'équipements & 14.5 & 27 \\
\hline Horlogerie/bijouterie & 20.5 & 32.5 \\
\hline Secteur tertiaire & $\mathbf{1 4}$ & $\mathbf{2 1 . 5}$ \\
\hline Hôtellerie-restauration & 20.5 & 28.5 \\
\hline Secteur secondaire et tertiaire & 15 & 23 \\
\hline
\end{tabular}

Note : La pénurie de main-d'œuvre qualifiée est mesurée à partir de questions sur les besoins en personnel qualifié et la difficulté ou non à les combler. L'excédent de main-d'œuvre non qualifiée est mesuré sur la base d'une question portant sur le nombre jugé suffisant, excédentaire ou manquant de personnel non qualifié.

Source : Office fédéral de la statistique. Indicateurs du marché de l'emploi 2005.

l'avantage aux spécialistes disposant d'un large savoir-faire dans un domaine restreint, mais aux généralistes avec une large formation de base leur permettant de comprendre un processus et de $s$ 'adapter rapidement à de nouveaux domaines. La principale mission de la formation des apprenti-e-s sera dorénavant d'encourager, en plus des bases techniques, des qualifications comme l'indépendance, la flexibilité, le sens de la responsabilité, l'aptitude à travailler en groupe et à communiquer, ainsi que la volonté d'apprendre. $»^{8}$

Ces trois domaines, par leur ancrage historique dans une culture ouvrière, par leur importance en matière d'emplois, mais aussi par la diversité de leurs caractéristiques, nous apparaissent comme étant de bons indicateurs de la situation et des évolutions que connaîtra la majorité de la population ouvrière dans les années à venir`.

\footnotetext{
${ }^{8}$ Centre professionnel du Nord-Vaudois, La formation de Polymécanicien, 2005.

${ }^{9}$ Une remarque s'impose néanmoins sur cette distinction entre branches. Quiconque se lance sur la voie d'une analyse par domaines d'activité se heurte rapidement, en Suisse, à l'obstacle que représente le manque de données statistiques détaillées. Ce manque d'information nous a souvent obligé à nous satisfaire de tendances élaborées à partir d'indicateurs qualitatifs relevés dans nos enquêtes. Il nous a également empêché d'aller aussi loin que nous l'aurions souhaité dans une analyse différenciée des trois domaines d'activité. Le lecteur voudra bien tenir compte de cette difficulté à la lecture de cet article, qui peut sembler présenter quelques lacunes en termes statistiques pour qui n'est pas au fait de la situation des sources fédérales.
}

\section{Une hétérogénéité de fait}

Sur l'ensemble des ouvriers et ouvrières qualifiés interrogés au cours de nos recherches, le plus grand nombre avait suivi un apprentissage officiellement reconnu qu'ils avaient réalisé juste après la fin de leur scolarité. Ils étaient donc détenteurs, dans leur majorité, d'un certificat fédéral de capacité. Parmi les types d'apprentissage, l'apprentissage dual, associant formation en entreprise et formation en école, dominait largement. Ce premier constat n'est guère surprenant. Il confirme une certaine convergence entre politique de formation et politique de recrutement.

La présence de plusieurs autres types de formation l'est davantage. Parmi eux, des formations étrangères, notamment des CAP français en cuisine, mécanique ou horlogerie. La question du statut de ces ouvriers qualifiés d'origine étrangère est un sujet en soi que nous n'aborderons pas dans le cadre de cet article. Il est néanmoins important de garder à l'esprit qu'une partie des difficultés de recrutement ont été comblées par l'engagement d'ouvriers étrangers, notamment de frontaliers, ouvrant largement le débat sur l'équivalence des diplômes. Cette question constitue un autre aspect de l'évolution des processus de qualification.

Nous y trouvons aussi des personnes ayant suivi un rattrapage en cours d'emploi, selon les dispositions de l'ancien article 41, ainsi que des personnes non diplômées, avec une formation acquise « sur le tas ». 
On relève enfin la présence de personnes détentrices d'un CFC, mais dans une autre profession que celle dans laquelle elles étaient occupées au moment de l'enquête. Celles-ci, présentes surtout dans l'horlogerie et la fabrication de machines, s'étaient reconverties grâce à une formation accélérée interne à l'entreprise.

Ce premier survol nous apporte trois informations importantes. D'une part, une fidélité au principe général de l'apprentissage et du CFC comme base du recrutement des ouvriers qualifiés et une certaine stabilité du recrutement de personnes possédant ce niveau de qualification. Nous n'avons pas rencontré de diplômés d'écoles supérieures.

D'autre part, si à première vue nous pouvions nous attendre à une homogénéité des formations, c'est plutôt à un constat d'une certaine hétérogénéité que nous avons été confrontés; signe s'il en est d'une certaine souplesse dans l'application des principes de base. Les formations « sur le tas » ou via l'article 41 sont plutôt classiques et bien connues, alors que la reconversion de personnes issues d'autres professions témoigne plutôt d'une tendance nouvelle.

Enfin, les données disponibles sont insuffisantes pour établir si des différences significatives existent entre les branches. Mais nous pouvons néanmoins mentionner quelques nuances: une présence plus importante de personnes sans formation diplômante dans la restauration et l'horlogerie et une moindre présence des procédures de reconversion dans la restauration.

La suite de cet article va nous permettre d'analyser plus en détails la nature de ces nouvelles filières et les enjeux associés à leur présence dans ces secteurs.

\section{LA FILIÈRE INFORMELLE EN DÉCLIN ?}

L'accès à la qualification par la seule formation « sur le tas », sans formation officielle, bien qu'encore présent sur le terrain, n'est plus guère à l'ordre du jour, comme le laissent penser les propos des ouvriers et de leurs employeurs. Ainsi un mécanicien, luimême diplômé, relevait que dans les années 60 « il y avait des gens qui étaient très habiles sur les machines, même en n'ayant pas fait d'apprentissage, ça devenait des spécialistes qui étaient capables de faire le travail comme un homme avec certificat. Parce qu'avec les années, ils s'étaient fait la main comme on dit». Il ajoute qu'aujourd'hui ce type d'ouvrier a pratiquement disparu.

Un horloger, formé sur ce même principe, et qui au fil des années est devenu l'un des meilleurs ouvriers de l'entreprise, relevait lui que son parcours « aujourd'hui, c'est de moins en moins [possible], parce que maintenant tous les jeunes arrivent avec un papier, j'entends avec un certificat ».

Dans le même esprit, une ouvrière sans formation initiale ni titre reconnu, qui réalisait un CFC d'horlogère selon l'article 41 au moment de l'étude, déclarait que le suivi d'une formation officielle était devenu pour elle le seul moyen de pouvoir accéder à des postes plus intéressants : «Je n'osais pas demander ou je me disais que comme ils nous appellent ouvrières petites mains, je n'avais pas de qualifications, donc je n'osais pas non plus trop demander. »

Aucune statistique n'existe sur l'évolution du nombre de personnes qui occupent des postes qualifiés sans détenir de CFC mais, au vu des exemples ci-dessus, tout porte à croire que pour ces deux domaines d'activités, leur nombre est plutôt en régression. L'évolution du rapport entre qualifiés et non-qualifiés (tableau 3) au sein de ces deux branches va en tout cas dans le sens d'un recrutement de plus en plus centré sur des personnes diplômées.

La situation est moins claire dans le domaine de la restauration. Dans l'organisation où nous avons mené nos observations, une institution publique spécialisée dans la restauration collective, l'ensemble des critères d'accès à l'emploi et aux catégories salariales venait d'être revu. La détention du CFC avait été rendue nécessaire pour prétendre à un poste d'ouvrier qualifié et au salaire correspondant. Plusieurs personnes, des femmes notamment, qui avaient pour tout bagage une formation «sur le tas » - l'une d'entre elle était même responsable d'une petite équipe - venaient d'être déclassées et replacées au rang de simple personnel d'exploitation (ouvrières non qualifiées). Jusqu'à cette décision très récente, une expérience acquise dans l'entreprise 
pouvait suffire pour être reconnu comme qualifié. Il semblerait que cette tendance à la hausse des qualifications ne se retrouve pas dans le cadre de la restauration privée (cafés, restaurants indépendants) qui accorde au contraire encore une large place à la formation "sur le tas", voire l'encourage, faute de trouver suffisamment de personnel diplômé. L'importance du nombre de non-qualifiés dans la branche, associée au très fort taux de pénurie de qualifiés, va dans ce sens. La convention collective de l'hôtellerie précise d'ailleurs que sous le terme de travail qualifié : «on entend une activité ou fonction régulière dans un domaine ou partie de domaine habituellement menée ou occupée par des professionnels, ou que l'on ne peut qualifier de travail subalterne. Dans le domaine de la cuisine, cette définition inclut les collaborateurs sans formation professionnelle qui préparent ou élaborent des mets dont la réalisation est généralement de la compétence d'un cuisinier ou d'un pâtissier. $»^{10}$ Elle admet ainsi clairement l'exercice d'un travail qualifié par quelqu'un qui n'en aurait pas le titre. La restauration semble donc présenter une évolution un peu différente de celles des deux autres domaines.

Tableau 3

Évolution des effectifs de la population travaillant dans les trois branches étudiées selon la qualification

\begin{tabular}{|l|c|c|c|c|c|c|c|c|c|c|}
\hline & \multicolumn{4}{|c|}{1990} & \multicolumn{5}{c|}{2000} \\
\hline & $\begin{array}{c}\text { Effectifs } \\
\text { totaux }\end{array}$ & $\begin{array}{c}\text { Sans } \\
\text { formation }\end{array}$ & $\begin{array}{c}\text { Appren- } \\
\text { tissage }\end{array}$ & $\begin{array}{c}\text { Formations } \\
\text { supérieures }\end{array}$ & $\begin{array}{c}\text { Sans } \\
\text { indication }\end{array}$ & $\begin{array}{c}\text { Effectifs } \\
\text { totaux }\end{array}$ & $\begin{array}{c}\text { Sans } \\
\text { formation }\end{array}$ & $\begin{array}{c}\text { Appren- } \\
\text { tissage }\end{array}$ & $\begin{array}{c}\text { Formations } \\
\text { supérieures }\end{array}$ & $\begin{array}{c}\text { Sans } \\
\text { indication }\end{array}$ \\
\hline $\begin{array}{l}\text { Construction } \\
\text { de machines }\end{array}$ & 143759 & $27 \%$ & $54 \%$ & $18 \%$ & $1 \%$ & 106214 & $19.5 \%$ & $50 \%$ & $27.5 \%$ & $3 \%$ \\
\hline Horlogerie & 30502 & $44 \%$ & $44.5 \%$ & $10 \%$ & $1.5 \%$ & 26678 & $34 \%$ & $41 \%$ & $19.5 \%$ & $5.5 \%$ \\
\hline $\begin{array}{l}\text { Hôtellerie- } \\
\text { restauration }\end{array}$ & 157410 & $43.5 \%$ & $42.5 \%$ & $9.5 \%$ & $4.5 \%$ & 176661 & $38 \%$ & $36.5 \%$ & $18.5 \%$ & $7 \%$ \\
\hline
\end{tabular}

Note : Ce tableau n'établit pas de distinction en fonction des postes occupés; il concerne tous les emplois de ces secteurs, encadrement compris. Il ne permet pas de savoir à quel type de poste sont affectées les personnes disposant de formations supérieures.

Source : Office fédéral de la statistique.

\section{LES FILIÈRES OFFICIELLES ALTERNATIVES SE MULTIPLIENT}

Plusieurs des ouvriers et ouvrières rencontrés avaient profité des possibilités de rattrapage qu'offrait l'ancien article 41. La suppression de cet article dans la nouvelle loi n'a pas supprimé toutes les possibilités d'acquérir officiellement un diplôme autrement que par la formation initiale, au contraire même. L'article 41 a été remplacé par plusieurs dispositions reconnaissant des filières « alternatives » à l'apprentissage officiel, notamment les formations dites

${ }^{10}$ Convention collective nationale de travail pour les hôtels, restaurants et cafés, 1 er juillet 2005 , art. 10. ch. 2 . modulaires $^{11}$ ou les procédures de validation d'acquis.

L'article 9 de la loi stipule par exemple que «les expériences, professionnelles ou non, la formation spécialisée et la culture générale acquises en dehors des filières habituelles sont dûment prises en compte ». L'ordonnance sur la formation professionnelle du 19 novembre 2003 fixe, quant à elle, l'organisation et les responsabilités pour la prise en compte des acquis : «Les cantons veillent à assurer des services de consultation chargés d'aider les

11 Par formation modulaire, il faut entendre une formation dont le contenu est découpé en différents modules qui peuvent être suivis chacun de manière séparée selon un mode de progression défini. La réussite de chaque module est liée à la réussite d'un examen. Le CFC est décerné lorsque l'ensemble des modules a été suivi et réussi. 
personnes à dresser l'inventaire des qualifications dont elles peuvent se prévaloir et qu'elles ont acquises en-dehors des filières de formation habituelles, à travers une expérience pratique, professionnelle ou non.» (Art 4). La nouvelle loi ouvre donc la voie à toute une série de nouvelles possibilités de formation.

La branche horlogère n'avait pas attendu ces nouvelles dispositions pour mettre en place une formation modulaire en horlogerie. Elle fait même figure de pionnière dans ce domaine. Dès 1994, elle introduit cette possibilité pour répondre aux besoins de cette industrie et au chômage persistant. Le métier d'horloger est décomposé en six modules distincts qui peuvent être acquis de manière séparée. Chaque module correspond à une activité exercée dans l'horlogerie et peut donc être mis en valeur et négocié directement dans les entreprises. Les lieux de formations sont décentralisés tout le long de l'arc jurassien dont un à Morteau. La formation se veut en effet transfrontalière ${ }^{12}$. Le suivi de l'ensemble des modules donne droit au CFC (ou au CAP français). Les cours sont dispensés le soir et le samedi ou à plein temps. En douze ans, 1076 personnes ont participé à cette formation et 48 ont accédé au CFC. 30 sont aujourd'hui en voie de terminer le dernier module, ce qui portera à près de 80 le nombre de CFC d'ici 2008. Le nombre de personnes concernées reste, on le constate, relativement faible par rapport au nombre d'apprentis (près de 200 par année) qui suivent la filière de l'apprentissage officiel. Cette filière demeure donc pour l'instant relativement marginale.

Toujours dans la même branche, un travail pilote en matière de reconnaissance de l'expérience acquise en entreprise pour les ouvriers sans qualification a été mené entre 1998 et 2001 (Marti, 1999 et 2003). Ce projet a abouti à ce que figure, au sein de la convention collective, l'obligation pour l'employeur de tenir à jour régulièrement une fiche de formation pour chaque employé-e, intégrant les formations suivies et les expériences acquises dans l'entreprise, ce document devant servir de base à une validation officielle des acquis.

${ }^{12}$ On notera ici la résolution par ce moyen de la question évoquée plus haut de l'équivalence des diplômes entre pays.
Cette procédure de validation des acquis introduite dans la loi en est aujourd'hui au stade de la formalisation à l'échelle fédérale. Le principe retenu, et susceptible d'évoluer encore, est basé sur un triple niveau de reconnaissance. Un niveau de reconnaissance individuelle qui correspond pratiquement à un bilan de compétences individuel, tel qu'il peut être pratiqué dans un centre de bilan. Il donne lieu à un portfolio de compétences.

Le second niveau, dit de reconnaissance institutionnelle, est une procédure au cours de laquelle «une instance officielle atteste que la personne a réellement fait preuve de l'acquisition de certaines compétences dans des domaines spécifiques. Cette forme de reconnaissance repose sur une démarche de reconnaissance personnelle complétée par une évaluation externe $»^{13}$. Elle conduit à une attestation de qualifications. Enfin le niveau de validation des acquis, qui consiste à établir le lien entre les acquis non-formels et les exigences liées à des certifications officielles (CFC par exemple) conduit à l'attribution de tout ou partie d'un diplôme reconnu. L'évaluation est faite de manière externe sous le contrôle direct des instances qui délivrent les diplômes (associations professionnelles, Confédération, etc.)

Ce système est testé depuis l'an 2000 dans le canton de Genève. Durant l'année 2004 par exemple, 357 personnes ont été admises en procédure de validation d'acquis. Les professions concernées figurent dans le tableau 4. À titre de comparaison, le canton de Genève forme annuellement environ 1900 apprenti-es, toutes professions confondues.

Bien qu'il soit encore difficile de mesurer l'impact réel de ces nouvelles formes d'acquisition de la qualification, leur présence constitue d'ores et déjà une source incontestable de diversité, et cela à plusieurs niveaux : dans les types de filières (avec les options modulaires ou de validation), dans le déroulement (avec les possibilités de découper la formation et de l'étaler sur plusieurs années) et dans les niveaux de qualification atteints (certification par module, en fonction du degré d'expérience, etc.). Ces options s'éloignent du principe de l'apprentissage

${ }^{13}$ Tiré de Association Valida (2006), Un système suisse de reconnaissance et de validation des acquis, guide des bonnes pratiques, p. 4 . 
Tableau 4.

Nombre de candidats à une validation d'acquis dans le canton de Genève selon la profession (2004)

\begin{tabular}{|l|c|}
\hline \multicolumn{1}{|c|}{ Profession } & Nombre \\
\hline Employé-e de bureau et de commerce & 162 \\
\hline Vendeur-euse et gestionnaire de vente & 77 \\
\hline Informaticien-ne & 24 \\
\hline Hôtellerie-restauration (cuisinier, sommelier, assistant d'hôtel) & 15 \\
\hline Assistante dentaire & 14 \\
\hline Nettoyeur-euse en bâtiment & 12 \\
\hline Maçon & 11 \\
\hline Assistante en information documentaire & 4 \\
\hline Gestionnaire en logistique & 4 \\
\hline Monteur électricien & 4 \\
\hline Horlogerie (horloger, polisseur) & 2 \\
\hline Divers & 28 \\
\hline Total & 357 \\
\hline
\end{tabular}

Source : rapport annuel 2005 du Centre de bilan de Genève.

classique et de la dichotomie non moins classique entre qualifiés et non-qualifiés, puisque, désormais, la loi autorise des niveaux intermédiaires de formation entre la qualification et la non-qualification. L'introduction de ces possibilités se veut une réponse aux « besoins toujours plus différenciés du monde du travail » (Message du Conseil fédéral, 2000, p. 5279). Elles restent néanmoins largement inscrites dans le cadre d'une certification officielle, celle des métiers existants, et continuent à être placées sous la surveillance collective des acteurs habituels. On ne peut donc parler, à ce stade, de réelle rupture avec le processus de qualification de base, mais plutôt d'un assouplissement.

\section{UNE NOUVELLE FILIÈRE NON OFFICIELLE ?}

À ces procédures en voie d'officialisation, il faut ajouter un autre phénomène plutôt nouveau. Comme nous l'avons vu plus haut, les entreprises horlogères et de fabrication de machines n'hésitent pas à favoriser la reconversion de personnes détentrices d'un
CFC dans une autre branche. Ces personnes sont intégrées dans la production moyennant une formation interne dont la durée peut s'échelonner de quelques mois à plus d'une année. Ainsi, typographes, boulangers, bouchers, cuisiniers ou autres se retrouvent dans la profession d'horloger ou de mécanicien. Il n'est pas possible de chiffrer l'ampleur de ces recrutements, mais ils ne sont manifestement pas négligeables. Dans l'entreprise observée, les candidats étaient par ailleurs plus nombreux que les places disponibles. Cela témoigne de l'existence d'un phénomène nouveau dans nos sociétés : le changement beaucoup plus fréquent de profession au cours d'une vie, que cela soit pour des raisons économiques (chômage) ou personnelles (désintérêt pour une profession, handicap, recherche de meilleures conditions, déménagement, etc.). Indice de ces évolutions : le taux de changement de profession est passé, entre 1970 et 2004 , de $38,9 \%$ à $45,4 \%$ dans les professions avec apprentissage. Les professions de l'ingénierie, de l'industrie de transformation et de production atteignent même le taux le plus élevé, tous niveaux de formation confondus $(46,9 \%)^{14}$. Ce

${ }^{14}$ Source : OFS - Enquête suisse sur la population active, 2004. 
n'est donc pas un hasard si ce phénomène se retrouve surtout dans l'horlogerie et la fabrication de machines. L'extrait d'entretien qui suit illustre bien ce type de nouveaux parcours fort peu linéaires : «J'ai déjà un $C F C$, donc à la base je suis mécanicien sur voitures. L'automobile ne m'a pas trop attiré. J'ai assez vite abandonné en fait. J'ai fait l'apprentissage, mais je n'ai jamais vraiment pratiqué tel quel le métier. J'y suis revenu plus tard, mais entre deux, j'ai fait des études de musique. Et puis, après ces études de musique, je suis revenu un moment dans l'automobile et comme cela ne me plaisait toujours pas [...] Il y a eu aussi des aléas, chômage, etc., reprendre le métier tel quel c'était un peu difficile. Il y a eu aussi des problèmes physiques, de genou. Et c'est en collaboration avec le chômage [Agence pour l'emploi] et aussi l'assurance invalidité que j'ai été orienté vers l'horlogerie. [...] Et puis l'horlogerie cela représentait une bonne possibilité. Alors c'est pour cela que j'y suis venu. J'ai demandé à faire cette formation ici pendant dix-huit mois. J'ai fait un stage, j'ai été accepté et je l'ai faite et j'ai réussi l'examen et voilà. Maintenant, j'ai à peu près un statut qui correspond à un $C F C$ de trois ans dans l'horlogerie. »

L'introduction de ce type de formation contribue ici encore à diversifier les filières de qualification, cette fois dans une pure logique d'entreprise, hors de toutes négociations collectives et hors de toute structure reconnue. Ces recrutements ont suscité des réactions parfois violentes de la part des ouvriers issus de la profession qui y voyaient une menace pour le métier. Un ancien typographe reconverti dans la mécanique y fait allusion :

Question: "Vous dites que vous avez eu une formation en 4 mois ?»

Réponse: " 4 mois c'est un peu une invention de l'entreprise ça. Au départ, il paraît qu'ils ont été beaucoup critiqués, parce qu'il y a des externes qui leur disaient "ouais ça va former des opérateurs CNC comme ça en 4 mois c'est pas possible, il faut du métier". »

Un mécanicien diplômé va dans le même sens : «Ils ont fait une erreur à mon avis quand ils ont engagé tout ce monde, ils ont engagé des bouchers, des boulangers qui n'avaient aucune formation [...] je dis c'est pas logique. J'ai toujours dit, ils veulent nous le casser le métier. »

Le statut de ces ouvriers qualifiés n'est pas clairement établi. L'ancien typographe cité plus haut avait de fait occupé plusieurs postes autrefois considérés comme semi-qualifiés (tournage, fraisage, rectifiage). De même, plusieurs horlogers engagés sous un régime comparable effectuaient des travaux un peu moins complexes que leurs collègues ou reconnaissaient avoir une connaissance un peu moins approfondie du métier. Manifestement, on pourrait donc les retrouver en concurrence directe avec les personnes issues de formations modulaires ou d'un processus de validation d'acquis.

La restauration se distingue ici encore des deux autres branches, puisque cette option y semble moins présente. $\mathrm{Au}$ contraire, on retrouve plutôt dans l'horlogerie et la fabrication de machines des ouvriers ayant abandonné la restauration, comme ce fut le cas de cet horloger: "Avant, j'étais dans l'hôtellerie puisque j'ai fait un apprentissage de pâtissier-confiseur, apprentissage de cuisinier après un cours accéléré de serveur et puis pour terminer le cours de cafetier. L'horlogerie, c'était complètement inconnu à mes yeux. Nous sommes partis à l'étranger avec mon épouse et puis nos trois enfants, on a travaillé pour une chaîne internationale d'hotellerie et puis étant donné que j'avais pas de suite de contrat immédiat, on ne pouvait pas se permettre de rester sans travailler à l'étranger avec trois enfants, donc on a décidé de rentrer en Suisse. Et puis, c'est là que je me suis rendu compte qu'en plusieurs endroits l'hôtellerie en Suisse, comparée à l'étranger, avait de grandes difficultés et puis elle ne payait pas ou plus comme il y a quelques années [...] et puis également en ce qui concerne la vie de famille qui devenait aussi assez difficile avec les enfants. C'est là que j'ai appris qu'il y avait la possibilité de faire un stage de 3 jours à titre d'information pour l'horlogerie, j'ai fait ces 3 jours. » Conditions de travail difficiles, faiblesse des salaires, instabilité d'un secteur, ces raisons poussent les professionnels à renoncer à ce métier après quelques années et expliquent peut-être la difficulté de la branche à y attirer des personnes issues d'autres professions. 
VERS UNE QUALIFICATION DE SECONDE ZONE ?

Il est bien évidemment trop tôt aujourd'hui pour tirer un bilan de ces nouvelles expériences. Un premier constat s'impose néanmoins quant à la participation à ces nouveaux dispositifs.

Les CFC d'horloger décernés par le biais de la formation modulaire représentent une moyenne annuelle d'environ 1 à $2 \%$ des CFC en horlogerie. Quant à ceux décernés à Genève dans le cadre de la validation des acquis, il est encore trop tôt pour en faire le compte. Si l'on admet, perspective pour le moins optimiste, que tous les candidats acceptés dans ce processus accèdent au diplôme, on atteindra au maximum $5 \%$ des titres délivrés en formation à Genève, soit le taux moyen qui existait sous le régime de l'article 41. Des résultats non négligeables certes, mais qui représentent néanmoins une proportion très restreinte de la population en formation et a fortiori de la population non qualifiée. Ces filières restent pour l'instant plutôt marginales. Quant aux formations maison, nous ne disposons d'aucune indication quant à l'importance de leur développement. Impossible en tout cas de parler actuellement d'un véritable renversement de la logique de l'apprentissage classique.

Quel que soit le développement futur de ces filières, une question se pose pourtant dès maintenant quant au statut dans l'entreprise de ces nouveaux diplômés. Les premières statistiques disponibles pour la formation modulaire en horlogerie le montrent : la majorité des personnes n'atteint pas le certificat final. La validation des acquis admet également, et par définition, des niveaux et des contenus de validation très différents. Quel sera dès lors le statut au sein des entreprises de ces personnes qui n'auront pas achevé l'ensemble de la formation puisque le CFC reste la référence? La question se pose au moins à deux niveaux. D'abord en matière salariale. La convention collective de travail des industries horlogères et microtechniques suisses ouvre peut-être une brèche à ce sujet. Si le principe général reste la distinction entre «travailleurs qualifiés et travailleurs non qualifiés », elle stipule aussi que «des salaires minimaux d'embauche pour d'autres catégories de personnel peuvent êtres introduits au niveau des cantons ou des régions, d'entente entre les associations patronales concernées et le secrétaire FTMH intéressé $»^{15}$. C'est admettre quelque part la possibilité d'une double échelle de salaire, selon le type de formation suivi. La convention de l'industrie des machines va plus loin, le salaire est attribué selon «La fonction, la prestation personnelle et la responsabilité individuelle $\gg .{ }^{16}$ Elle n'intègre pas de distinction, du moins formellement, entre les types de qualification, préférant une prise en compte de l'emploi et des prestations individuelles. Si elle accorde une plus grande marge d'intégration pour les personnes, comment cette approche se marie-t-elle avec un soutien réaffirmé par ailleurs à l'apprentissage ? Les observations faites en entreprise laissent plutôt penser que le titre reste encore une référence implicite très forte et que la prestation individuelle ou la nature du poste sont plutôt des éléments complémentaires dans la détermination du salaire.

Enfin, la classification adoptée dans le cadre de l'entreprise publique pour ses employés de cuisine fonde la distinction des classes sur la détention du $\mathrm{CFC}$ et n'accorde ainsi que peu de place à des formations intermédiaires. La convention de l'hôtellerie privée n'offre guère plus d'ouverture. Tout au plus mentionne-t-elle la possibilité d'une formation équivalente au CFC dans ses échelles de salaires.

Statut parallèle, individualisation potentielle, rattachement à un statut de non-qualifiés, les échelles salariales actuelles n'offrent pas nécessairement une place bien définie à ces nouveaux diplômés qui échappent aux références habituelles.

La question se pose aussi quant au type de poste occupé. L'exemple d'un ouvrier de l'industrie des machines suscite la réflexion. Après s'être reconverti dans la profession de dessinateur sur machines, il est engagé dans l'entreprise de machines comme magasinier, poste considéré longtemps comme non qualifié. L'extrait qui suit montre à la fois l'impossibilité pour cette personne d'accéder à un poste correspondant à ses qualifications, mais en même temps l'augmentation des exigences associées au

\footnotetext{
${ }^{15}$ Convention collective de travail des industries horlogères et microtechniques suisses du $1{ }^{\text {er }}$ janvier 2002, art. 14.2.4

${ }^{16}$ Convention dans 1'industrie des machines du 1er juillet 2003 au 30 juin 2008, art. 14 .
} 
poste occupé qui le rend de plus en plus inaccessible à une personne sans qualification.

Question: "Mais là, vous n'utilisez finalement pas ce que vous avez appris?»

Réponse: "Ah pas du tout, sauf bon dans le sens que...»

$\mathrm{Q}$ : «Vous savez ce que sont les pièces... »

$\mathrm{R}$ : « Voilà, exactement, il y a des dessins. C'est clair que pour chaque pièce il y a un dessin, je peux juste m'amuser à regarder, c'est seulement pour le souvenir quoi. Et puis bon c'est clair que dans la position où je suis, c'est intéressant [d'avoir une formation]. Je connais bien les états de surface par exemple, je sais ce que ça veut dire. Les magasiniers qui ont 15-20 ans [d'entreprise] quand ils [la direction] ont mis au point les contrôles de qualité, là ça a commencé à devenir un peu tendu [pour eux] dans le sens que c'est un peu des inconscients et puis ils n'ont pas d'éthique professionnelle. Mais ils ont toujours travaillé comme ça jusqu'à maintenant. [...] Moi je suis magasinier, bon je préférerais dessiner, d'ailleurs c'est ça que j'ai fait et puis malheureusement je travaille dans une entreprise que ça concerne à $100 \%$, mais je n'ai jamais eu l'opportunité. J'ai essayé déjà de travailler dans le bureau technique, c'est clair. Deux ou trois fois, ils cherchaient quelqu'un dans l'entreprise, mais cela ne passe pas. »

La pleine reconnaissance du niveau de formation par l'entreprise n'est pas garantie, sans pour autant que le poste occupé soit tout à fait celui d'un non-qualifié, et le risque de voir émerger, au travers de ces formations, une catégorie de qualifiés de « seconde zone» n'est pas à exclure. D'autant plus que la reconnaissance des pairs n'est pas acquise elle non plus. Les réactions les plus virulentes rencontrées dans le cadre de l'expérience de reconnaissance des acquis dans l'horlogerie émanent des ouvriers qualifiés : Est-ce que ces personnes ont vraiment la même formation, est-ce qu'elles acquièrent vraiment les mêmes capacités ? La méfiance des pairs est importante.

C'est dire combien l'intégration de ces nouveaux formés sur le marché du travail et dans les entreprises reste aujourd'hui sujette à caution.

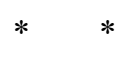

$*$
Le processus adopté par la Suisse en matière de qualification ouvrière, en apparence fidèle au principe indéfectible de l'apprentissage, ne manque pas, comme on vient de le voir, d'être traversé par des mouvements qui le remettent en partie en question et en modifient les contours. Comme le relevait C. Paradeise, la «fermeture d'un marché du travail n'est jamais définitivement acquise. Elle est le résultat incertain d'activités qui cherchent à transmuer les activités contingentes de l'environnement en nécessité sociale ». Elle ajoute que jusqu'à un certain point «c'est de sa solidité et de sa souplesse notons que ce second trait peut entretenir un rapport contradictoire avec le premier - face aux fluctuations de l'ensemble de son système de ressources et de contraintes que dépend sa capacité de reproduction » (Paradeise, 1988, p. 17).

Les orientations prises ces dernières années en matière de qualification ouvrière, en tout cas dans les trois domaines observés, montrent bien ce constant mouvement de reconfiguration, impliquant à chaque fois des formes de renégociation entre les acteurs.

C'est en fait un double mouvement qui vient aujourd'hui remettre en cause le processus. L'augmentation des exigences qualitatives imposée par des modes de production de plus en plus contraignants et par des positionnements sur des marchés de pointe, à haute valeur ajoutée, a conduit à repenser la qualification dans le sens d'un resserrement autour du titre officiel qu'est le certificat fédéral de capacité. L'apprentissage est présenté comme la base par excellence de la formation ouvrière, et l'attestation formelle de suivi que représente le titre s'impose désormais comme gage de qualité et comme référence pour la reconnaissance de la qualification. Cette formalisation accrue des filières marque un renforcement du principe professionnel. Le diplôme étant l'émanation d'un compromis négocié au sein des professions entre les différents partenaires, il ne laisse plus guère d'ouverture vers d'autres formes d'apprentissage (notamment informel) liées à l'entreprise.

Ce mouvement entre néanmoins en contradiction partielle avec un état du marché de l'emploi marqué par la pénurie endémique de main-d'œuvre qualifiée 
et un chômage persistant, voire une mobilité interprofessionnelle plus importante. Cette situation conduit les entreprises, comme les institutions étatiques, à trouver des solutions pouvant d'un côté répondre au besoin et de l'autre favoriser l'emploi des chômeurs. Dans les exemples observés, les entreprises n'hésitent pas à recruter hors de la profession et à bousculer la définition de la qualification en introduisant des formations maison. En même temps, les politiques de réinsertion dont sont issues la plupart des dispositifs de validation des acquis contribuent à introduire une diversité plus importante dans les filières d'accès aux titres reconnus. Si ces politiques de réinsertion et de validation ont été pensées dans le cadre des systèmes de formation officiels, il n'est pas certain pour autant qu'elles n'entraînent pas à court terme une révision des principes de base, notamment lorsqu'il s'agira de résoudre la question du statut des personnes qui n'auront atteint que des niveaux de formation partiels.

Dans l'un comme dans l'autre cas, les solutions adoptées introduisent une certaine diversité qui repose soit sur une plus grande individualisation des formations (modules, validation des acquis), soit sur un recentrage sur l'entreprise (formation maison).

Ces aspects ne manquent pas de heurter directement les défenseurs d'un processus strictement professionnel, comme en témoignent les réactions d'ordre corporatiste rencontrées au cours de nos recherches. L'un des enjeux du débat dans les prochaines années sera donc d'observer comment ces deux mouvements, potentiellement contradictoires, seront intégrés et renégociés par les acteurs sociaux. Ces derniers, comme on a pu le voir dans les trois domaines d'activité étudiés, élaborent des solutions propres à leur branche. Se dessine ainsi un engagement plutôt axé sur la formation des non-qualifiés pour l'horlogerie, alors que la restauration pourrait s'acheminer vers le maintien plus classique d'une voie reposant sur l'expérience. L'horlogerie et la fabrication de machines ont également exploré les possibilités de la reconversion de personnes déjà qualifiées, ce qui est moins le cas dans la restauration.

Les options en matière de politique salariale sont aussi très diverses, se rattachant explicitement à une hiérarchie classique dans le cadre de l'horlogerie et de la restauration, plus empreintes d'une logique d'entreprise dans la fabrication de machines. Sousjacentes à ces différences, on voit poindre les spécificités culturelles, sociales, techniques ou économiques propres à chacun de ces domaines, spécificités qui mériteraient un examen approfondi. Quoi qu'il en soit, les solutions seront multiples, reflétant l'autonomie relative dont jouit chaque profession.

Pour autant, l'enjeu des débats en cours ne se limite pas à la seule mesure de la capacité d'adaptation d'un processus. Ces débats sont aussi porteurs d'enjeux sociaux non négligeables, notamment pour les personnes les moins qualifiées. Un resserrement strict sur la détention du diplôme pourrait priver ceux-ci d'une voie d'accès à la qualification jusquelà privilégiée, alors que rien ne permet de savoir aujourd'hui si les nouvelles filières formelles seront investies par ce public. Mises au point pour lui, le risque qu'elles passent à côté de leur cible n'est pas absent, comme ce fut le cas de l'article 41 . Si tel devait être le cas, la catégorie des non-qualifiés se trouverait de fait exclue de toutes les possibilités d'accéder à une qualification dans un contexte où la présence de nouveaux candidats (les diplômés d'autres professions) se positionnent comme concurrents directs. On pouvait penser, ces vingt dernières années, que la question n'en serait bientôt plus une, compte tenu de la baisse significative du nombre de personnes sans qualification (de $45 \%$ en 1980 à $32,8 \%$ en 2000 selon le recensement de la population). À y regarder de plus près, les perspectives ne sont peut-être plus aussi claires. D'une part, le taux de non-qualifiés, après une baisse importante dans les années 80 , tend à se stabiliser depuis dix ans autour de $30 \%$. D'autre part, si jusqu'aux années 2000 les contingents de non-qualifiés appartenaient aux tranches d'âge les plus élevées, le taux de nonqualifiés a explosé aujourd'hui chez les jeunes de moins de 25 ans (entre 40 et $50 \%$ selon les enquêtes). Les enjeux ne sont donc pas moindres puisqu'il y va de l'intégration ou de la marginalisation définitive des ouvriers les moins qualifiés, mais peut-être désormais et surtout des plus jeunes.

Si le recours à ces nouvelles filières devait s'intensifier, une autre question pourrait devenir importante : 
le statut des nouveaux formés. Rien ne permet aujourd'hui de déterminer sous quelle forme ces personnes seront intégrées dans les entreprises et quelle sera leur place sur le marché de l'emploi. Le maintien du certificat fédéral de capacité (CFC) comme référence centrale limite largement toute approche diversifiée. Ne serions-nous pas, dès lors, sur le point d'officialiser cette ancienne catégorie très floue des «semi-qualifiés » et par là même de rigidifier d'anciennes hiérarchies entre qualifiés ? Cela alors même que le but initial de ces nouvelles filières était plutôt d'apporter une certaine souplesse. L'enjeu est ici encore de taille, puisqu'il pourrait s'agir de la production officiellement reconnue d'un système de qualification à deux vitesses. Le débat à ce sujet ne fait que commencer...

\section{Bibliographie}

Conseil Fédéral, Message du Conseil fédéral relatif à une nouvelle loi sur la formation professionnelle $d u$ 6 septembre 2000, Berne.

Dubs R. (2006), Rapport d'expertise sur les questions concernant la formation professionnelle en Suisse, Hep Verlag, Berne.

FNRS (2004), « Participation et non-participation à la formation continue professionnelle. L'exemple des horlogers et horlogères ainsi que des cuisiniers et cuisinières. », Synthèse $\mathrm{n}^{\circ} 5$, Téléchargeable sur : www.nfp43.unibe.ch

Marsden D. (1989), Marchés du travail et marchés de l'emploi, Économica, Paris.

Marti L. (2003), “Didn't you say Unqualified? On the job training and qualification processes", in Dybbroe B, Ollagnier E. (dir.), Challenging gender in lifelong learning: European perspectives, Roskilde University Press, p. 193-207.

Marti L. (1999), Femhor. Étude exploratoire pour une reconnaissance et une validation de l'expérience acquise dans l'industrie horlogère, FTMH/SMUV, Berne.
Marti L., Messant F., Modak M. (2005), Vies de mécaniciens, Éditions Antipodes, Lausanne.

Maurice M., Sellier F., Silvestre J. (1982), Politique d'Éducation et Organisation industrielle en France et en Allemagne, Puf, Paris.

Méda D. et Vennat F. (dir.) (2004), Le travail non qualifié. Permanences et paradoxes, La Découverte, Paris

Paradeise C. (1988), «Les professions comme marchés du travail fermés », in Sociologie et Sociétés, vol. XX, n², p. 9-21

Schraeder-Naef R. (dir) (1997), Warum Erwachsene (nicht) lernen?, Rüegger, Coire/Zurich.

Schraeder-Naef R., Fromm R.-J. (2003), Auswirkungen der Nachholbildung auf die berufliche Weiterentwicklung der Absolventinnen und Absolventen am Beispiel des Lehrabschlusses nach Artikel 41.1 $B B G$, Rapport de recherche, PNR 43.

Tabin J.-P. (1989), Formation professionnelle en Suisse. Histoire et actualité, Éditions Réalités sociales, Lausanne. 


\section{Résumé}

\section{La qualification ouvrière en Suisse: vers un système à deux vitesses?}

Laurence Marti

Depuis cinquante ans, la Suisse a opté pour un processus de qualification ouvrière de type professionnel, centré sur l'apprentissage. Que devient aujourd'hui ce processus confronté à l'augmentation des exigences qualitatives en matière de formation, à la pénurie de main-d'oeuvre qualifiée et au chômage persistant? Cet article fait un état de la situation, des évolutions en cours, ainsi que des enjeux sousjacents, en s'appuyant sur les études menées par l'auteur dans trois branches d'activités différentes : la restauration, I'horlogerie et la fabrication de machines.

\section{Mots clés}

Ouvrier qualifié, Apprentissage, Enseignement technique-professionnel, Suisse

Journal of Economic Literature: M 53 Training ; J 82 Labor force composition 


\section{POUR N 195 - OCTOBRE 2007}

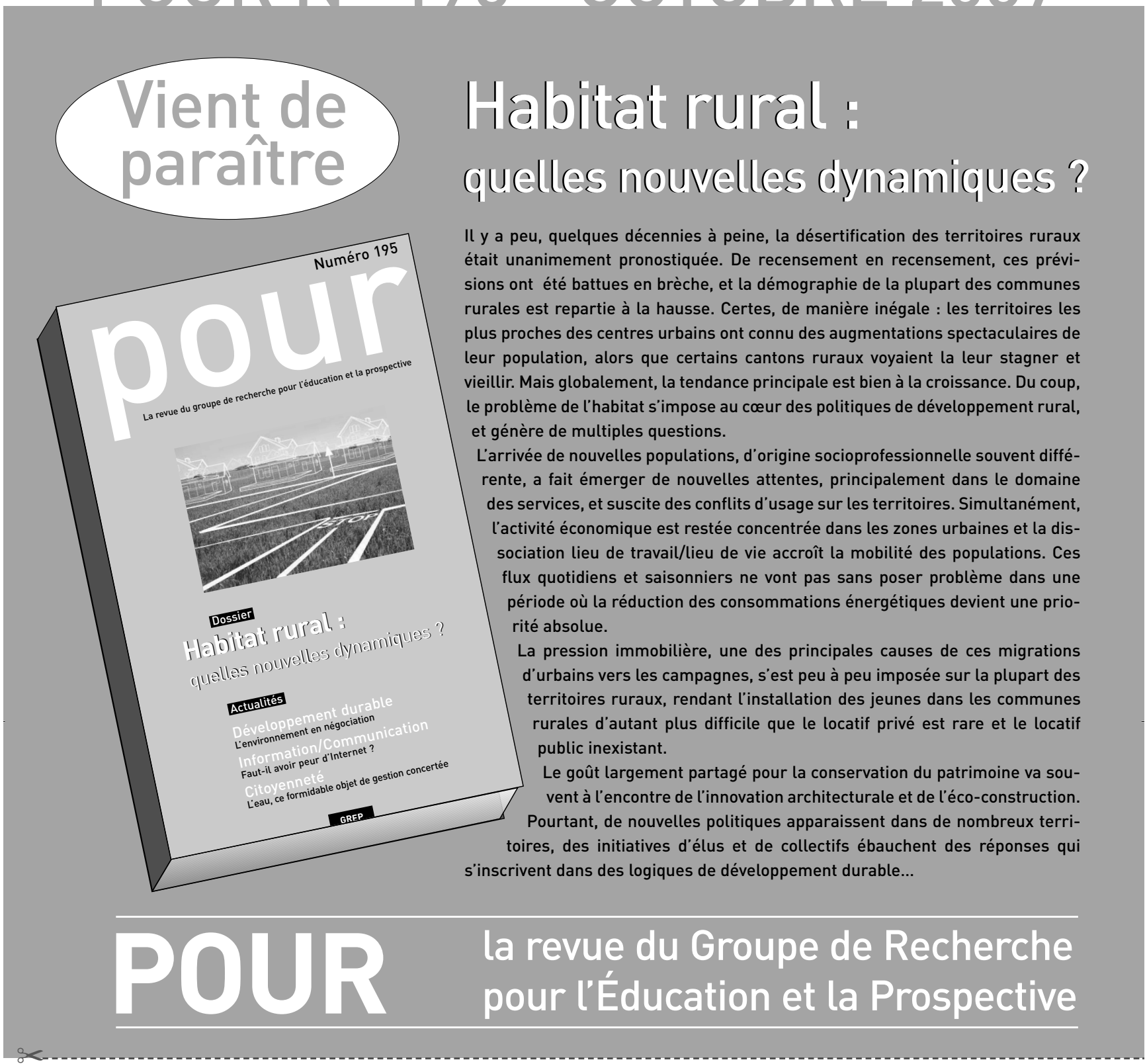

Revue POUR n 195 - octobre $2007-20 €$

Joindre $\mathbf{3} €$ pour participation aux frais de port et emballage
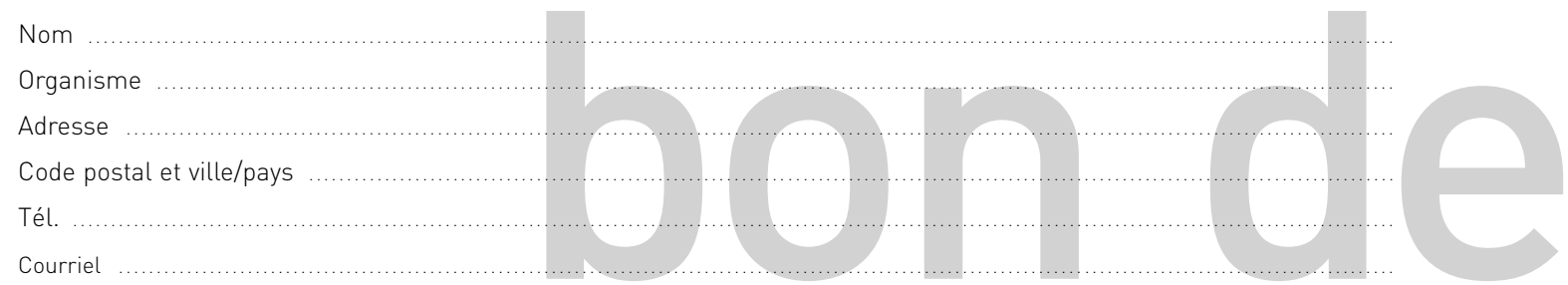

Abonnement 2007 (4 numéros) France $64 €$ - Étranger 73,20€

À retourner au GREP - 60/62, rue du Faubourg St-Martin - 75010 PARIS - Tél. : 0155331040 - Fax : 0155331041 Courriel : grep.pourawanadoo.fr - Site : www.grep.fr
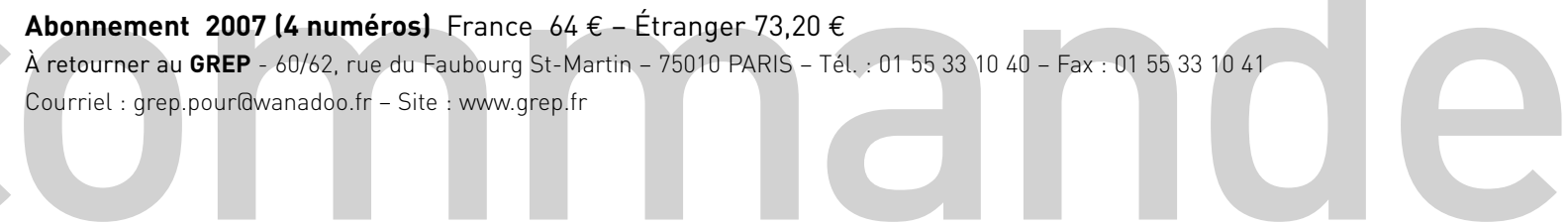